\title{
A New Approach to the Blind Deconvolution Problem Based on the Maximum Independency of Signals
}

\author{
Rongrong Wang ${ }^{1, a^{*}}$ and Fei $\mathrm{Xu}^{1, \mathrm{~b}}$ \\ ${ }^{1}$ Xijing university, Xi'an, China \\ a381713963@qq.com, b107803350@qq.com
}

Keywords: Blind deconvolution; Measure independence; Objective function; Seismic wavelet; Inverse filter

\begin{abstract}
In view of band-limited seismic data with a low dominant frequency and short data records, this paper proposes a new approach to the seismic blind deconvolution problem, a new criterion for blind deconvolution is constructed, by which we can measure the independency of output signals. The optimization program for new criterion of blind deconvolution is performed by applying Neidell's wavelet model to the inverse filter, which then makes the optimization program for multivariate reduce to univariate case. The proposed method can broaden the spectrum of seismic data effectively resulting in the improved resolution even in the case of band-limited seismic data with a low dominant frequency and short data records. The simulation test and actual seismic data processing results show that the correctness of the method.
\end{abstract}

\section{Introduction}

In seismic exploration, a recorded seismic trace is often represented as the result of a superposition of wavelets of constant shape weighted by the reflectivity series, thus it can be modeled as a convolution between the source signature and the reflectivity series. Deconvolution is an important process by which the reflectivity series can be estimated from the recorded trace, vertical resolution of the seismic image will be enhanced[1,2]. As the reflectivity series, the wavelet are unknown, only seismic trace is accessible, we have the classical blind deconvolution problem. Statistical deconvolution appears to be one of powerful tool for dealing with practical situation. Many blind deconvolution techniques, under various assumptions about the seismic wavelet and reflectivity series, have been developed with respect to different criteria in recent decades[3,4,5,6,7,8]. Anthony Larue[9] propose a new blind single-input single-output deconvolution method based on the minimization of the mutual information rate of the deconvolved output. To take the band limited nature of seismic wavelets and the presence of noise into account, Baan and Pham[10] present a modification of the mutual-information rate, whitening the deconvolution output only within the wavelet pass band, to prevent noise amplification, Wiener filtering is introduced.

In the above entropy-based approaches, one key point is to estimate the probability density function of the deconvolution output, however, estimating the PDF by nonparametric method needs certain length of the data to provide a good estimation. A seismic signal is treated to be stationary just within a limited time window due to intrinsic attenuation of the earth medium, thus these entropy-based approaches seem to result in a limited performance in the case of short data records.

In statistics theory, the mutual information is the one of measures to show the dependence between two continuous random variables. Based on Scarsini's work [11], we can see that concordance, as measures of dependence between two continuous random variables $X$ and $Y$, is a useful tool when dealing with blind deconvolution problem. The overall intention of this article is to construct a new criterion based on the concordance for seismic deconvolution, and We apply the wavelet model derived by N. S. Neidell to designing the inverse filter this wavelet model has two shape parameters. If we know the dominant frequency of the wavelet, only one parameter is left, which means the optimization program for multivariate reduces to univariate case without setting initialization and adding constraint to inverse filter. 


\section{Methodology}

Concordance and Measures of Concordance. Two observations $\left(X_{1}, Y_{1}\right)$ and $\left(X_{2}, Y_{2}\right)$ from a pair of continuous random variables are concordant, if $X_{1}<X_{2}$ and $Y_{1}<Y_{2}$, or $X_{1}>X_{2}$ and $Y_{1}>Y_{2}$; and they are discordant, if $X_{1}<X_{2}$ and $Y_{1}>Y_{2}$, or $X_{1}>X_{2}$ and $Y_{1}<Y_{2}$. Scarsini introduced a set of axioms for measures of concordance for pairs of random variables, which are invariant under as. increasing transformations of the random variables. When dealing with blind deconvolution problem, the following theorem of Scarsini may be considered.

Theorem. Let $\varphi$ be a bounded monotone odd function defined on $\left[-\frac{1}{2}, \frac{1}{2}\right]$, then $I(X, Y)$ satisfies all axioms for measures of concordance if

$$
I(X, Y)=\frac{\int_{0}^{1} \int_{0}^{1} \varphi\left(u-\frac{1}{2}\right) \varphi\left(v-\frac{1}{2}\right) d C_{X Y}(u, v)}{\int_{0}^{1} \varphi^{2}\left(u-\frac{1}{2}\right) d u}=\frac{E_{C_{X Y}}\left[\varphi\left(u-\frac{1}{2}\right) \varphi\left(v-\frac{1}{2}\right)\right]}{E_{M}\left[\varphi^{2}\left(u-\frac{1}{2}\right)\right]}
$$

Where $C_{X Y}$ is the copula of the real random variables $(X, Y)$. This theorem gives us a new way for constructing the new optimal criterion to deal with blind deconvolution problem.

On the Inverse Filter. Following the work by Baan and Pham, we can show that if the wavelet is known, Wiener filter achieves an optimum solution for inverse filter, since it makes a compromise between signal recovery and noise reduction. In the frequency domain, it can be written as

$$
A_{\text {opt }}(\omega)=\frac{\bar{W}(\omega)}{\mid W(\omega)^{2}+\varepsilon^{2}}
$$

Where $W(\omega)$ is the wavelet, the superscript of the numerator denotes the complex conjugate, $\varepsilon^{2}$ is a normal factor.

In the blind deconvolution context, the wavelet is unknown, and need to be estimated, therefore, we adopt N. S. Neidell's wavelet model to constructing inverse filter[12], which is derived from practical observation, and its $\mathrm{z}$ transform has the form.

$$
W_{N}(Z)=(1-Z)^{n}(1+Z)^{m} \quad n>0, m>0
$$

Furthermore, if we denote the peak frequency of the amplitude spectrum of this wavelet as $\omega_{p}$, the relationship between the $n$ and $m$ is

$$
\cos \omega_{p}=\frac{m-n}{m+n}
$$

So, if we know the peak frequency of the amplitude spectrum of real wavelet, only one parameter needs to be determined, in practice, usually we do not know this peak frequency; we have following value as a substitution for $\omega_{p}$

$$
\hat{\omega}_{p}=\underset{\omega}{\arg \max }\left|F T\left(x_{n}\right)\right|
$$

Where $F T$ denotes Fourier transform.

\section{New Deconvolution Algorithm}

Based on the above results, we construct a new frequency domain criterion corresponding to the inverse filter A for blind deconvolution. First, for the stationary seismic signal $\left\{x_{n}\right\}$, each sample of the trace has the same distribution functions, empirical distribution function can be applied to 
calculate the Cumulative distribution function $u_{n}$ corresponding to the sample $x_{n}$

$$
u_{n}=\frac{1}{N} \sum_{i=0}^{N-1} \mathbf{1}\left\{x_{i} \leq x_{n}\right\}
$$

Where $1\{A\}$ is the indicator function of event $A, N$ is the length of the data. Then the new criterion can be given as

$$
J=\left|E\left(\varphi\left(u_{n}-\frac{1}{2}\right) \varphi\left(u_{n+l}-\frac{1}{2}\right)\right)\right|
$$

$l$ denotes the time delay. After determining the $\omega_{p}, J$ is just the function of $n$ or $m$, so we have

$$
\begin{gathered}
n_{\text {opt }}=\underset{n}{\arg \min J(A)} \\
A_{\text {opt }}(\omega)=\frac{\bar{W}_{N}\left(n_{\text {opt }}, \omega\right)}{\mid W\left(n_{\text {opt }}, \omega\right)^{2}+\varepsilon^{2}}
\end{gathered}
$$

Thus our new deconvolution algorithm consists of the following steps:

- Estimate $\omega_{p}$ of the seismic data $\left\{x_{n}\right\}$.

- Compute the output $\left\{u_{n}^{n}\right\}$ of the inverse filter with the different parameter $n$, in which the superscript $n$ denotes the shape parameter of the inverse filter; $u_{n}$ is given by Eq.6, and the inverse filter is given by Eq.2 and Eq.3.

- For a suitable $\boldsymbol{l}$, compute the cost function $J$ corresponding to the shape parameter $n$ of the inverse filter.

- Where we let $\varphi$ equal. $x$.

- Search the minimum of the cost function to get the optimum inverse filter.

\section{Example}

Simulation Experiments. We have simulated the seismic trace by convolving a super-Gaussian reflectivity with a $40 \mathrm{~Hz}$ Ricker wavelet. The reflectivity is generated as $b^{3}(n)$, where $b(n)$ are independent normal random variables with zero mean and variance 0.08 . The sample interval of the synthetic trace is $1 \mathrm{~ms}$.
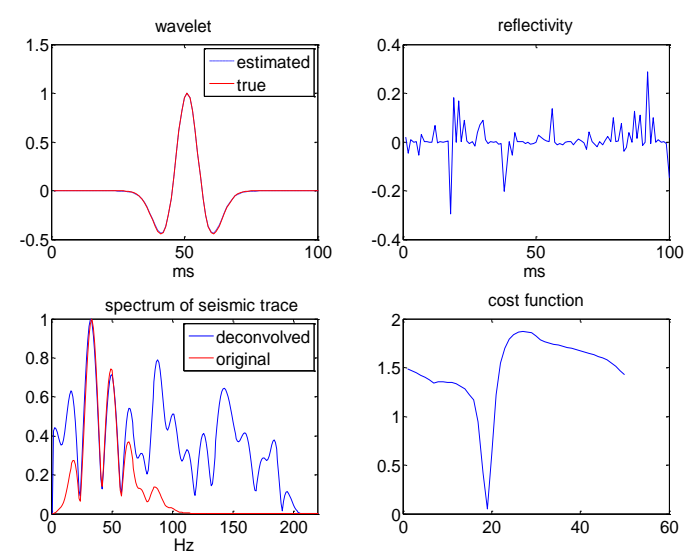

Figure 1. The results of deconvolution in the noise-free case.
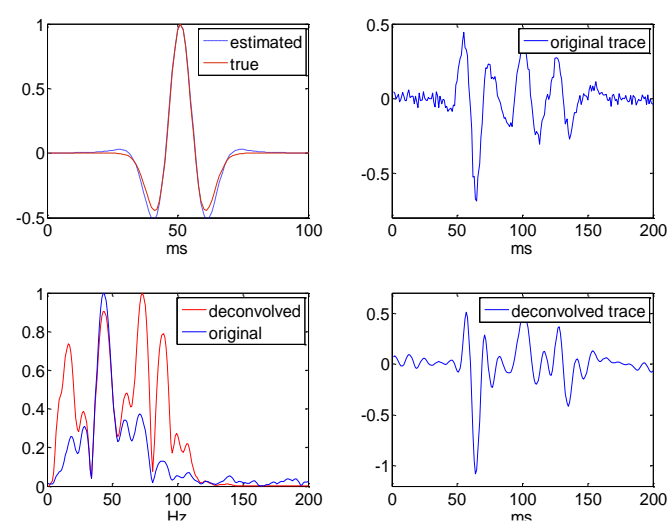

Figure 2. The results of deconvolution for a noisv svnthetic 
In order to assess the performance of our method for the data with small samples, we set the length of the synthetic trace about $200 \mathrm{~ms}$. The estimation results are plotted in Fig. 1. As shown in the figures, the wavelet (blue line) is very precise estimated. The original spectrum (red) ranges 0 $\mathrm{Hz}$ to $100 \mathrm{~Hz}$, and the deconvolved spectrum (blue) range is $0 \mathrm{~Hz}$ to $200 \mathrm{~Hz}$. The spectrum of the deconvolved trace is obviously broadened.

To test our algorithm for noisy signals, we employed a noisy synthetic trace. The signal-to-noise ratio of the noisy data is $15 \mathrm{~dB}$. The results are shown in Fig. 2. The wavelet (blue line) is well estimated. The original spectrum (blue) ranges $0 \mathrm{~Hz}$ to $80 \mathrm{~Hz}$, and the deconvolved spectrum (red) range is $0 \mathrm{~Hz}$ to $100 \mathrm{~Hz}$. The spectrum of the deconvolved trace is definite broadened. Meanwhile, the result of the deconvolved trace shows the higher signal-to-noise ratio, which demonstrates an anti-noise feature of our method.

Actual Data. We applied our method to a real onshore single seismic data, which was obtained from the Ordos Basin, China. As shown in Fig. 3, the resolution of the deconvolved seismic trace is clearly improved. The original spectrum (blue line) ranges $0 \mathrm{~Hz}$ to $120 \mathrm{~Hz}$, and the deconvolved spectrum (red line) ranges $0 \mathrm{~Hz}$ to $180 \mathrm{~Hz}$. In contrast with the spectrum of the original trace, the spectrum of the deconvolved trace is obviously broadened in Fig. 4.
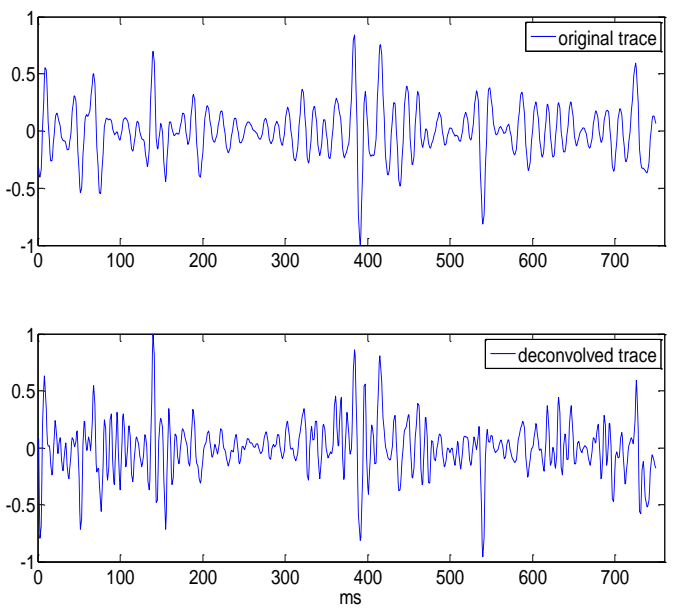

Figure 3. The comparison in the waveform before and after processing

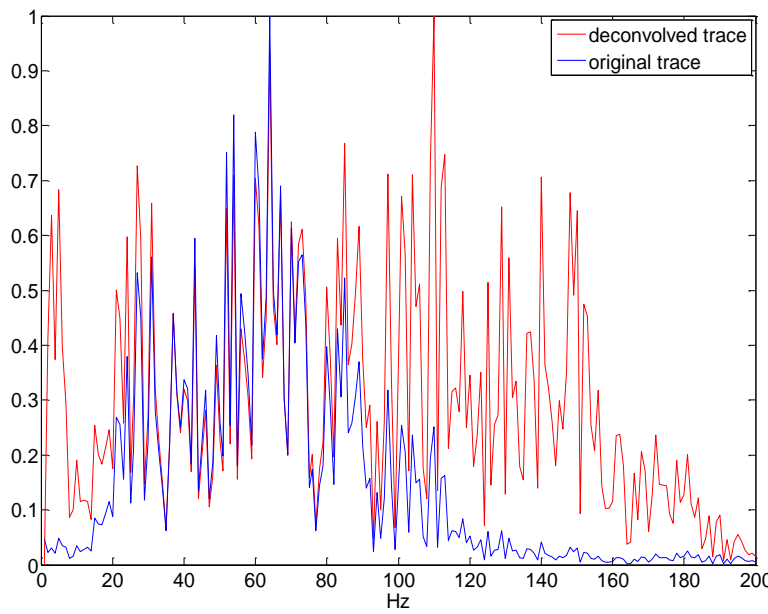

Figure 4. The comparison in the amplitude spectrum before and after deconvolution

An offshore stacked section is to assess the potential of applying our method. The stacked section was obtained from China. There are 50 traces in this seismic section. The sampling interval of data is $1 \mathrm{~ms}$. Fig. 5 displays the offshore data and result after deconvolution using our method. The resolution of the deconvolved section is clearly improved. 


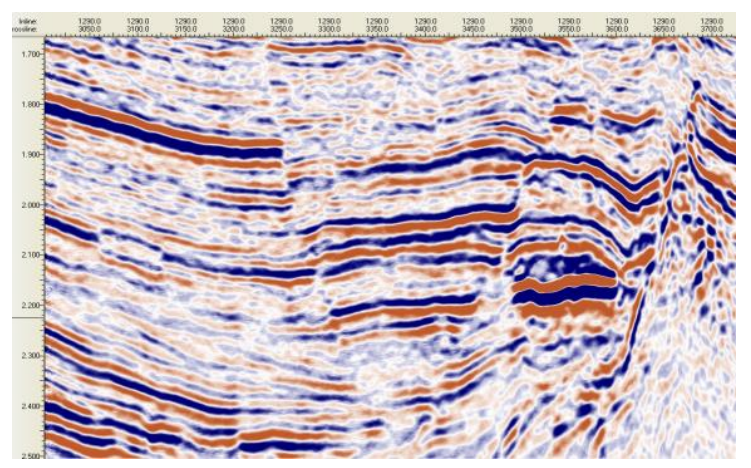

(a) Original record

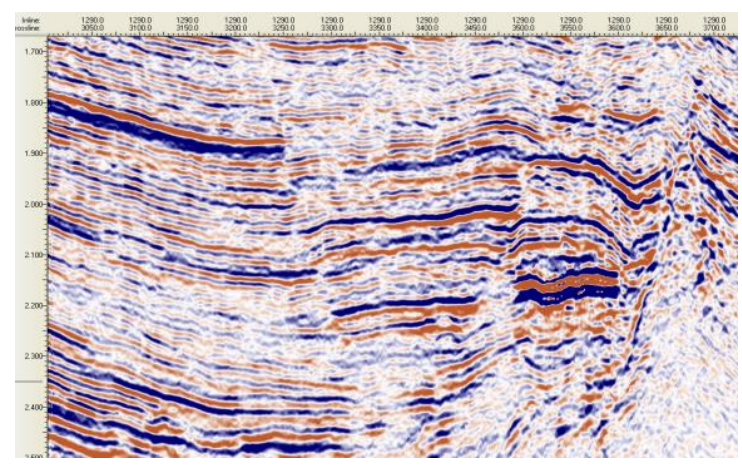

(b) Processing result

Figure 5. Compared before and after processing

\section{Summary}

A new blind deconvolution algorithm is proposed in this paper, whicn is based on the independence measurement of the random variables. The new algorithm can adopt the maximization criterion of independence to finish the deconvolution processing of the signal in a relatively short data records. Furthermore, Both the simulation calculation and the actual data processing have confirmed the correctness of the theory. In the practical application, the theory can be used in the non-stationary seismic signals processing, which can obtain a good effect.

\section{Acknowledgement}

Scientific Research Program Funded by Shaanxi Provincial Education Department (Program No.2013JK1121).

\section{References}

[1] X.W. Liu and H. Liu: Progress in Geophysics, Vol. 18 (2003) No.2, p.203.(In Chinese)

[2] H.L. Wang, Z.Y. Ding, D.J. Gui, et al: Geophysical prospecting for petroleum, Vol. 52 (2013) No.1, p.49.(In Chinese)

[3] C.Y. Wu, R.J. Yang, Z. Bao, et al: Geophysical prospecting for petroleum, Vol. 48(2009)No.3, p.232.(In Chinese)

[4] J.H. Gao, M. Zhang and B. Zhang: Process in geophysics, Vol. 27 (2011) No.2, p.540.(In Chinese)

[5] B. Zhang, J.H.Gao: Entropy, Vol. 13(2011) No.9, p.1730.

[6] H.S. Li,G.C. Wu and X.Y. Yin: Process in geophysics, Vol. 27(2012) No.3, p.0936.(In Chinese)

[7] L.F. Call and X.M. Tian: Process in geophysics, Vol. 27 (2012) No.3, p.1116. (In Chinese)

[8] W Gao: Blind Deconvolution based on Independent component analysis Abstract(Ph.D., Ocean University of China, China 2011), p.11.(In Chinese)

[9] Larue, J.I. Mars and C. Jutten: IEEE transactions on signal processing, Vol. 54(2006) No.5, p.1771.

[10]M.V. Baan and D.T. Pham: Geophysics, Vol. 73 (2008) No.5, p.37. 
[11]M.Scarsini: Stochastica, VIII (1984) p.201.

[12]N. S. Neidell: Geophysics, Vol. 56(1991) No.5, p. 681. 